\title{
Prevalencia real de Epilepsia en un Programa de Salud Mental. Merida-Venezuela
}

Real prevalence of epilepsy in a mental health programme in Merida, Venezuela

Juana Rondón ${ }^{1}$, Xiomara Betancourt ${ }^{2}$ y Alba Contreras ${ }^{2}$

1 Departamento de Fisiopatología, Facultad de Medicina. Universidad de Los Andes. Mérida-Venezuela.zucamzucam280@hotmail.com

2 Programa de Salud Mental. Corporación de Salud. Mérida, Venezuela

Recibido 4 Febrero 2008/Enviado para Modificación 16 Agosto 2008/Aceptado 4 Septiembre 2008

\section{RESUMEN}

Objetivos Esta investigación tuvo como objetivos: 1. Determinar precisión del diagnóstico de epilepsia entre Programa Salud Mental y la evaluación neurológica experta, en población rural del Estado Mérida; 2. Conocer la frecuencia de epilepsia según variables demográficas.3. Determinar el tipo de crisis epiléptica mas frecuente.

Métodos Se aplicó un cuestionario y evaluación por neurólogos de la Clínica de Epilepsia-IAHULA, entre Marzo-Diciembre 2003 a la población previamente diagnosticada con epilepsia, por el programa de Salud Mental, en el medio rural.

Resultados De 240 pacientes evaluados, se corroboró diagnóstico de epilepsia en 198, correspondiendo a 82,3 \% de validez del diagnóstico. El grupo etario con mayor frecuencia fue de 0-44 años, con un pico entre 15-24 años; respecto a clasificación de crisis epilépticas: generalizadas (53,5\%), crisis focales con generalización secundaria $(30,8 \%)$.

Conclusiones Se obtuvo cifras elevadas de epilepsia, (precisión diagnóstica de 82,3 $\%$, respecto al Programa Salud Mental); estos pacientes no recibían atención neurológica, ni el beneficio de estudios complementarios pertinentes. El conocimiento de estos resultados, ha puesto en evidencia la necesidad del recurso neurológico para el Programa de Salud Mental, con el objeto de brindar una verdadera atención integral, al paciente con epilepsia en el estado Mérida.

Palabras Clave: Epilepsia, prevalencia, salud mental (fuente: DeCS, BIREME).

\section{ABSTRACT}

Objectives Determining the accuracy of the mental health programme in diagnosing epilepsy and expert neurological evaluation in rural populations in the state of Mérida in Venezuela and ascertaining the most frequent types of epilepsy in the population being investigated.

Methodology Neurologists from the IAHULA epilepsy clinic applied a questionnaire throughout May and December 2003 and evaluated the rural population previously diagnosed as suffering from epilepsy by the mental health programme. 
Results A diagnosis of epilepsy was confirmed in 198 of the 240 patients evaluated (82,3\% diagnostic accuracy). Epilepsy was most frequent in the 0-44 age-range, peaking between 15-24 years. Epileptic seizures were classified as being generalised $(53,5 \%)$ or focal seizures having secondary generalisation $(30,8 \%)$.

Conclusions Very high levels of epilepsy were obtained (82.3\% diagnostic accuracy compared to the mental health programme); however, these patients did not receive neurological care or benefit from complementary studies. These results revealed the need for neurological resources for the mental health programme for providing comprehensive care for epilepsy patients in Mérida.

Key Word: Epilepsy, prevalence, seizure, mental health (source: MeSH, NLM).

L a epilepsia dentro de la práctica neurológica, constituye uno de los más frecuentes motivos de consulta; las cifras de prevalencia en países en vías de desarrollo son bastante elevadas, difiriendo notablemente de las observadas en países desarrollados (1-26). En nuestro país y mas específicamente en el estado Mérida, se ha determinado a través de investigaciones clínico epidemiológicas, que la epilepsia es altamente prevalente $(27,28)$, de allí ha surgido el interés hacia la epilepsia, y el deseo de prestar adecuada atención al paciente con esta entidad, de la necesidad observada en el trabajo diario de la consulta especializada de la Unidad de Neurología del Instituto Autónomo Hospital Universitario de Los Andes (I.A.H.U.L.A.).

El Programa de Atención Integral al paciente con epilepsia, diseñado en 1999 y aplicado parcialmente, persigue la asistencia especializada (terciaria) y la cobertura en el medio rural, mediante la implementación de un sistema de atención semejante a las Clínicas Móviles de Epilepsia, trasladándose así el recurso especializado al lugar de origen de la población, con el objeto de mejorar de esta forma los índices de cobertura y deserción $(29,30)$. En nuestra región a través del Programa de Salud Mental (PSM) (31), se ha llevado la atención psiquiátrica al medio rural, haciendo el psiquiatra del programa, supervisión y atención directa a través de visitas programadas a los diferentes centros ambulatorios rurales. En los últimos cinco años, le ha correspondido atender a los pacientes con epilepsia en estas áreas (por la elevada presentación de este trastorno y por no contar con recurso neurológico). En el PSM se obtuvieron cifras estadísticas de epilepsia alarmantes, alertando a la coordinación de la Clínica de Epilepsia del Instituto Autónomo Hospital Universitario de Los Andes (IAHULA), que ha diseñado el Programa de Atención Integral al paciente con epilepsia.

Es por ello que se realizó esta investigación vinculando la atención terciaria (IAHULA) y la atención primaria en el medio rural (PSM), con el objeto de 
corroborar el diagnóstico de epilepsia en estas poblaciones atendidas por el psiquiatra.

Los objetivos del estudio fueron: 1-Determinar la precisión en el diagnóstico de epilepsia entre el PSM y la evaluación neurológica experta, en la población rural del estado Mérida- Venezuela y 2- Conocer el tipo de crisis epiléptica mas frecuente en la población investigada.

\section{MÉTODOS}

Durante los meses de Marzo y Diciembre 2003, se realizó entrevista y evaluación neurológica, a la población previamente diagnosticada con epilepsia por el personal del Programa de Salud Mental (PSM). Se corroboraron las cifras proporcionadas por el Programa de Salud Mental y se realizaron visitas a la cabecera de los Distritos Sanitarios Tovar, Mucuchíes, Lagunillas y El Vigía del estado Mérida. La población de los diferentes distritos sanitarios del estado Mérida, guarda características socioeconómicas semejantes; en líneas generales la actividad económica preponderante es la agropecuaria y solamente en la cabecera de los distritos sanitarios la actividad económica proveniente de servicios prestados, surge como de cierta importancia. Sólo en el Distrito Sanitario El Vigía existe una actividad económica mas elevada, proveniente de la ganadería y las industrias procesadoras de alimentos y del petróleo, pero la población atendida por el Programa de Salud Mental, no proviene de estos estratos socioeconómicos. Se procedió a la aplicación del Formato de Entrevista al Paciente con Epilepsia diseñado para esta investigación, basado en cuestionarios de epilepsia previamente validados (32,33), y en la historia clínica de epilepsia utilizada en IAHULA (29); el cuestionario fue aplicado a través de entrevistas por dos residentes de postgrado de neurología y dos neurólogos (uno de los cuales contaba con entrenamiento especializado en epilepsia) quienes realizaron la evaluación neurológica experta bajo la tutoría de la Clínica de Epilepsia del IAHULA.

Este personal se trasladó al medio rural, para evaluar a toda la población de pacientes identificados con epilepsia por el psiquiatra y/o el médico en ejercicio rural del programa de salud mental y que acudieron a la consulta neurológica. Los pacientes fueron previamente convocados, con suficiente antelación por los médicos de los ambulatorios rurales correspondientes a cada distrito. Se realizaron dos visitas por cada distrito, con 15 días de diferencia entre ellas. En caso de que existiera duda respecto al diagnóstico, el paciente era evaluado a través de una segunda entrevista por el neurólogo especializado en epilepsia, quien actuaba como árbitro determinando si se trataba de un cuadro de epilepsia o no lo era. 
Criterios de Inclusión:

1-Evaluación previa por médico en ejercicio rural y/o psiquiatra.

2-Diagnóstico previo de epilepsia.

Criterios de Exclusión:

1-Edad fuera del límite establecido.

2-Sin evaluación previa.

3-Sin diagnóstico de epilepsia o con otro diagnóstico neurológico y/o psiquiátrico.

En la investigación, no se utilizaron exámenes complementarios (electroencefalograma, tomografía computarizada y resonancia magnética), debido a la dificultad que tenía la población para asistir a centros especializados como el IAHULA, donde existen estos recursos. Y debido a las dificultades socioeconómicas de la población y a que el PSM tenía limitada disponibilidad de drogas antiepilépticas, tampoco se contempló el régimen terapéutico, para correlacionar con los diferentes tipos de crisis epilépticas.

\section{RESULTADOS}

Fueron evaluados 240 pacientes, distribuidos por distrito sanitario de la siguiente manera: El Vigía 89 pacientes (37,1 \%), Tovar 68 pacientes (28,3 \%), Mucuchíes 52 pacientes (21,7 \%), Lagunillas 31 pacientes (12,9\%). Se corroboró el diagnóstico de epilepsia en 198 pacientes valorados (82,3\% de concordancia en el diagnóstico).

En relación con el rango etario se evaluaron pacientes desde 6 meses hasta 67 años de edad, observándose un predominio en la presentación de la enfermedad, entre las edades de $0-44$ años (88,4\%), con un pico entre $15-44$ años, con $66,2 \%$.

Con respecto al género, se evaluaron 104 (52,5 \%) pacientes del género femenino, y 94 (47,5\%) del género masculino, lo que hace la población estudiada bastante homogénea en cuanto a género.

En cuanto a las características semiológicas de las crisis epilépticas, se pudo observar que en los pacientes estudiados por el grupo de neurólogos, hubo predominio de crisis epilépticas generalizadas (106 pacientes-53,5\%), seguidas de las crisis epilépticas focales secundariamente generalizadas (61 pacientes-30,8 \%) y las crisis focales en último lugar (31 pacientes- 15,7 \%). No se hizo 
correlación con la clasificación de las crisis epilépticas, realizado por el PSM según categorías, ya que en ocasiones no se discriminaba el tipo de crisis del paciente y se hacía sólo diagnóstico de epilepsia en forma general.

Respecto al tratamiento recibido por los pacientes debe hacerse la observación, que la elección de la droga antiepiléptica (DAE), no se hacía según el tipo de crisis; pero como se mencionó previamente, el PSM proporcionaba la medicación (fenobarbital y difenilhidantoína mayormente), en ocasiones carbamazepina y en ningún caso valproato o acido valproico; esto hace probable que se indicara la DAE disponible para el momento, en el PSM. Por ello no se hace correlación, con este aspecto de la evaluación.

\section{DISCUSIÓN}

En esta investigación debe resaltarse que la población estudiada corresponde a un grupo de pacientes que pudiera considerarse "población cautiva” por poseer el diagnóstico de epilepsia asignado a través del PSM. Y que la búsqueda de precisión en el diagnóstico de epilepsia se ha logrado claramente al obtener cifras elevadas (82,3 \%) de la entidad, corroboradas en el diagnóstico por el personal especializado en Neurología. Aún cuando existe un 17,7 \% de error en el diagnóstico, para el PSM, debe tomarse en cuenta que quien hacía el diagnóstico inicial de Epilepsia, era el médico en ejercicio rural y el psiquiatra (sin especialización en el área de epilepsia) y en nuestro país corresponde el manejo de esta entidad al especialista en Neurología.

Es de destacar que la epilepsia, está afectando mayormente a las edades mas productivas de la vida, ya que hemos observado un pico importante entre los 15 y 44 años de edad y se trata de población que, como consecuencia de su enfermedad, pudiera estar fuera del sistema educativo y del aparato laboral, y además se desconoce el impacto sobre la fertilidad, y otros aspectos importantes para la calidad de vida $(34,35)$.

Dentro de la clasificación de crisis epilépticas, en la mayoría de estudios realizados (de prevalencia, incidencia o de frecuencia en series clínicas), se han obtenido cifras entre 45-65\%, mas elevadas en la categoría de crisis epilépticas focales, anteriormente denominadas parciales (36), a diferencia de nuestros resultados, en los que predominan las crisis epilépticas generalizadas 53,5\%, lo que podría hacer suponer de un substrato genético, ya que este tipo de crisis predomina en los síndromes epilépticos de índole hereditario (37). 
Nuestra investigación confirmó la existencia de cifras elevadas de epilepsia en el medio rural del estado Mérida, los cuales no estaban beneficiándose de atención neurológica especializada, ni de la realización de estudios complementarios pertinentes. Esto obliga a plantear la necesidad de asignación del recurso neurológico para el PSM, ya que existe un 17,7 \% de error en el diagnóstico que podría mejorarse, reubicando al personal especializado en psiquiatría, para atender la nosología psiquiátrica y no la neurológica. De igual modo el neurólogo asignado al PSM, podría determinar las consecuencias de las elevadas cifras de epilepsia, su impacto sobre la calidad de vida y de esta manera prestar una verdadera atención integral, al paciente con epilepsia en el estado Mérida •

\section{REFERENCIAS}

1. Shorvon SD, Farmer PJ. Epilepsy in Developing Countries: AReview of Epidemiological, Sociocultural, and Treatment Aspects. Epilepsia. 1.988; 29 (Suppl.1) 36-54.

2. Hauser WA, Kurland LT. The Epidemiology of Epilepsy in Rochester, Minnesota, 1.935 through 1.967. Epilepsia 1.975;16 1-66.

3. Hauser WA, Annegers JF, Kurland L. Prevalence of Epilepsy in Rochester. Minnesota. 1.9401.980. Epilepsia. 1.991; 32 429-445.

4. Shorvon SD. Epidemiology, classification, natural history, and genetics of epilepsy. Lancet. 1.990; 336 93-96.

5. Schoenberg BS. Recent Studies of the Epidemiology of Epilepsy in Developing Countries. A Coordinated Program for Prevention and Control. Epilepsia. 1987; 28: 721 - 722.

6. Rose SW, Penry JK, Markush RE, Radloff LA, Putnam PL. Prevalence of Epilepsy in children. Epilepsia. 1.973; 14: 133-152.

7. Osuntokum BO, Schoenberg BS, Nottidge VA, Adeuda A, Kale O, Adeyefa A, et. al. Research protocol for measuring the prevalence of neurologic disorders in developing countries. Results of a pilot study in Nigeria. Neuroepidemiology. 1.982; 1: 143-153.

8. Goodridge DMG, Shorvon SD. Epileptic seizures in a population of 6000. I. Demography, diagnosis and classification, and role of the hospital services. BMJ. 1.983; 287: 641-645.

9. Koul R, Rasdan S, MottaA. Prevalence and Pattern of Epilepsy (Lath/Mirgi/Laran) in Rural Kashmir, India. Epilepsia. 1.988; 29: 116-122.

10. Rwiza HT, Kilonzo GP, Haule J, Matuja WB, Mteza I, Mbena P. et. al. Prevalence and Incidence of Epilepsy in Ulanga, a Rural Tanzanian District: A Community-Based Study. Epilepsia. 1.992; 33: 1051-1056.

11. Attia N, Mrabet A, Hamida MB. Prevalence of Epilepsy in Kelibia, Tunisia. Epilepsia. 1.993; 34: 1038-1032.

12. Chiófalo N, Kirschbaum A, Fuentes A, Cordero M L, Madsen J. Prevalence of Epilepsy in Melipilla, Chile. Epilepsia. 1.979; 20: 261-266.

13. Escobedo F. Prevalence of Epilepsy in children of the Tlalpan District in Mexico City in 1975. México City, México. National Institute of Neurology (Ministry of Health and Welfare); 1975.

14. Placencia M. Shorvon SD, Paredes V, Cascante S, Sander JWAS. Epileptic Seizures in an Andean Region of Ecuador. (part a). Brain. 1.992; 115: 771-782.

15. García F, Rubio F, García F, Escobedo F, Goncalez A. Prevalence of epilepsy in children: Tlalpan, México City, México. Neuroepidemiology. 1.983; 2: 16-23.

16. Gómez JG, Arciniegas E, Torres J. Prevalence of epillepsy in Bogotá, Colombia. Neurology. 1.978; 28: 90-95.

17. Pradilla G, Puentes F, Pardo CA. Estudio Neuroepidemiológico piloto. Universidad Industrial de Santander. 1.984; 8: 133-139. 
18. Cruz ME. Barberis P, Enríquez J, Izurieta H, Bossano F, Proaño J etal. Estudios Neuroepidemiológicos en el Ecuador. Comité Ecuatoriano de Neuroepidemiología. Quito - Ecuador. Ministerio de Salud Pública;1.986.

19. Bielen I, Cvitanovic L, Bergman B, Kosicek M, Planjar M, Vuksic L, et al. Prevalence of epilepsy in Croatia: a population-based survey. Acta Neurol Scand. 2007; 116(6): 361-7

20. Melcon MO, Kochen S, Vergara RH. Prevalence and clinical features of epilepsy in Argentina. A community-based study. Neuroepidemiology. 2007; 28(1): 8-15.

21. Somoza MJ, Forlenza RH, Brussino M, Licciardi L. Epidemiological survey of epilepsy in the primary school population in Buenos Aires. Neuroepidemiology. 2005; 25(2):62-8.

22. Aydin A, Ergor A, Ergor G, Dirik E. The prevalence of epilepsy amongst school children in Izmir, Turkey. Seizure. 2002;11(6):392-6.

23. Sridharan R, Murthy BN. Prevalence and pattern of epilepsy in India. Epilepsia. 1999; 40(5):631-6.

24. Durá T, Yoldi ME, Gallinas F. Epilepsy in children in Navarre. An Sist Sanit Navar. 2007; 30(2): 20714.

25. Zarrelli MM, Beghi E, Rocca WA, Hauser WA. Incidence of epileptic syndromes in Rochester, Minnesota: 1980-1984. Epilepsia. 1999; 40(12): 1708-14.

26. Ponce P. Estudio de prevalencia de transtornos neurológicos en Lezama. Estudios Neuroepidemiológicos en Venezuela. Caracas-Venezuela. Departamento de Enfermedades Neurológicas. Ministerio de Sanidad y Asistencia Social. Venezuela; 1.985. p.16-38.

27. Rada R. Prevalencia de la Epilepsia en escolares de la ciudad de Mérida. Venezuela (1.975). Boletín de la Sociedad de Médicos Internos y Residentes. Hospital Universitario de los Andes. 1.983; 1; 1(2): 13-21.

28. Avanzini G, Beghi E, de Böer H, Engel J Jr, Sander JW, Wolf P. Epilepsia. En: Trastornos Neurológicos desafíos para la salud pública. Estados Unidos de América: Organización Mundial de la Salud; 2006.

29. Rondón J, Encinoza O. Programa de Atención Integral al paciente con epilepsia. Act Neurol Colomb. 1998; (Suppl. 48): 48-49

30. Watts AE. A model for managing epilepsy in a rural community in Africa. BMJ. 1.989; 298: 805-807.

31. Coordinación Regional de Salud Mental. Estado Mérida. Plan Regional de Salud Mental. Primera edición. Mérida-Venezuela. Corporación de Salud-Facultad de Medicina-U.L.A. 1.993. p.39.

32. Reutens DC, Howell A, Gebert KE, Berkovic SF. Validation of a Questionnaire for Clinical Seizure Diagnosis. Epilepsia. 1.992; 33 : 1065-1071.

33. Placencia M, Sander JWAS, Shorvon SD, Ellison RH, Cascante M. Validation of a screening questionnaire for the detection of epileptic seizures in epidemiological studies. Brain. 1.992; 115: 783-794.

34. Baker G. Assessment of Quality of life in People with Epilepsy: Some Practical Implications. Epilepsia 2001; 42 (Suppl 3): 66-69.

35. Devinsky O, Vickrey B, Cramer J, Perrine K, Hermann B, Meador K et al. Development of the Quality of Life in Epilepsy Inventory. Epilepsia. 1995. 36 (11): 1089 - 1104.

36. Serrano PJ, Olivares J, Guardado P. Caracterización epidemiológica de la epilepsia del adulto subsidiaria de ingreso hospitalario. Rev Neurol 2001; 32 (11): 1013-1019.

37. Berkovic SF, Scheffer I. Genetics of the Epilepsies. Epilepsia. 2001; (Suppl 5): 16-23. 